\title{
Enseñar periodismo para leer y narrar la sociedad del siglo XXI
}

\author{
Teach Journalism for Reading and Narrating the Society \\ of the 2Ist Century
}

(ARTÍCULO DE REFLEXIÓN)

JORGE MANRIQUE GRISALES. Pontificia Universidad Javeriana de Cali, Cali, Colombia. (jmanrique@javerianacali.edu.co)

- Recibido: 01/marzo/2012. Aceptado: 07/mayo/2012

\section{RESUMEN}

Este artículo de reflexión recoge algunas preguntas en torno al periodismo y su impacto en la sociedad. Se hace un rastreo de los debates que se han planteado en Latinoamérica desde escenarios como Felafacs, Claep, SIP, Fnpi y Unesco, entre otros. De igual forma, se confrontan distintas visiones desde la academia, los medios y los investigadores que indagan por el lugar del oficio de informar en el complejo mundo de la Comunicación y las Ciencias Sociales. Se exponen algunas propuestas y tendencias en la formación de periodistas, teniendo como retos los nuevos contextos sociales y la emergencia de los formatos asociados al uso de las TIC, el rol de los docentes, los componentes curriculares y los nuevos espacios de formación generados entre medios y academia.

Palabras clave: periodismo, prensa, comunicación y periodismo, enseñanza del periodismo, acreditación en periodismo, ciberperiodismo.

\section{ABSTRACT}

This is an article of reflection that collects some questions about journalism and its impact on society. A scan of the debates that have been raised in Latin America from scenarios such as Felafacs, Claep, SIP, Fnpi and Unesco, among others. Similarly, different visions are confronted from academy, media and researchers who investigate the place of the job to inform the complex world of communication and social sciences. Outlining some proposals and trends in the training of journalists with the challenges the new social contexts and the emergence of the formats associated with the use of ICT, the role of teachers, the curriculum components and new training spaces generated between media and academy.

Keywords: journalism, media, communication and journalism, journalism, accreditation in journalism education, cyberjournalism. 
“¿De qué le valen al periodismo todos los avances de la tecnología si al fin pierde su norte?". Javier Darío Restrepo.

"Los profesores de periodismo deben esforzarse por conjugar el pasado con futuro, en un entorno profesional que ya es presente". Ramón Salaverría.

Como objeto de formación, el periodismo plantea de entrada algunas preguntas no solo en cuanto a su lugar dentro del campo de la comunicación sino, y fundamentalmente, en lo que tiene que ver con su impacto en la sociedad. En la epidermis del debate salen a relucir temas como la concentración mediática, la calidad de los contenidos, la responsabilidad social de la prensa, la libertad de expresión y la ética. De fondo, aparece un incierto terreno abonado por varias disciplinas de las ciencias sociales, las humanidades y la lingüística, entre otras, en el que resulta difícil inscribir "al mejor oficio del mundo", como lo denominara Gabriel García Márquez. En esta compleja taxonomía se recuerda la voz de Kapuscinski (2003, p.18) quien decía que "el periodista es un cazador furtivo en todas las ramas de las ciencias humanas".

Desde varias miradas, posturas y propuestas, el presente artículo indaga por la relación entre comunicación y periodismo en América Latina. Posteriormente, repasa el tejido de un oficio construido en el tiempo desde distintos referentes culturales, sociales y académicos. Asimismo, de la mano de periodistas y académicos examina los giros del periodismo en la era de la información; los retos, las percepciones que del oficio tienen los recién egresados, así como las nuevas apuestas en materia de formación.

En síntesis, si la pregunta gira en torno al lugar del periodismo para leer y narrar la sociedad del siglo XXI, como se plantea en el título de este artículo, es necesario reflexionar sobre la caja de herramientas que ofrecen el oficio y las instancias desde donde se piensa, se diseña y se ejerce.

\section{MARCO TEÓRICO}

\section{ENTRE PERIODISMO Y COMUNICACIÓN}

En junio de 2005, la Fundación Nuevo Periodismo Iberoamericano (FNPI), con sede en Cartagena, Colombia, se hizo la pregunta “¿Hacia dónde va el periodismo?” y convocó a sus más ilustres maestros de todo el continente a debatir y tratar de dar respuesta a esta cuestión.

El planteamiento del problema tuvo, desde Germán Rey, cuatro aspectos a tener en cuenta en un intento por perfilar posibles respuestas a la pregunta: la crisis del periodismo vinculada a los cambios que experimentan las sociedades; la irrupción de las nuevas tecnologías; el perfil futurista de los medios y por último "una afirmación vocacional de las vicisitudes que ayer y hoy caracterizan al oficio" (CAF, 2008, p.14)

En el afán de fijarle un norte al periodismo, Pena de Oliveira (2009) plantea dos tipos de tensiones, una relacionada con la teoría y la práctica, y la otra con el lugar del periodismo en la comunicación, pensando, al igual que otros autores como Traquina (2004) y Souza (2008), en una eventual teoría del periodismo basada en dos objetivos básicos: “Por qué las noticias son como son? y ¿cuáles son los efectos que esas noticias generan?" (p.228).

Marques de Melo (1997) recuerda que Argentina y Brasil fueron los primeros países del continente que incluyeron al periodismo en el sistema universitario, en la década de los años 30. Vallejo (2006), precisa que en Colombia fue la Universidad Javeriana la primera en ofrecer estudios de periodismo en 1935.

Años después, en la década de los setenta, un proceso alentado por la Unesco, a través del Centro Internacional de Estudios Superiores de Periodismo para América Latina, Ciespal, propuso homogenizar los programas universitarios en periodismo bajo el rótulo de Comunicación Social. Para Marques de Melo (1997) "Ciespal** comete la equivocación de transformar las escuelas pioneras de periodismo en Facultades de Ciencias de la Información sin evaluar la coyuntura internacional y sin observar la naturaleza de los modelos vigentes en las sociedades capitalistas avanzadas" (p.126)

Como consecuencia de lo anterior, explica el mismo Marques de Melo, se crearon "guetos comunicológicos" al interior de las universidades, que en los años 80 "convirtieron las escuelas de Comunicación en fábricas de desempleados" (Marques de Melo, 1997, p.126)

Este planteamiento de Marques de Melo fue ratificado por Samper (2012), durante un videochat en el marco del Hay Festival de Cartagena 2012, al señalar que al periodismo "se le quiso poner corbata" al emparentarlo con la Comunicación Social, un tronco común del que también egresaban publicistas y relacionistas públicos:

Tengo la idea de que lo que siempre llamamos periodismo, en un momento dado se resolvió subirlo de estatus y ponerle corbata... Entonces se llamó Comunicación Social. Pero en aras de una discusión razonable, es evidente que hay otra serie de carreras como la publicidad y las relaciones públicas que podrían considerarse Comunicación Social y, por lo tanto, serían hermanas del periodismo. (Samper, 2012) 
Samper cuestiona la inclusión del periodismo dentro del campo de la Comunicación Social porque, según dice, "se renunció a los talleres y a otros elementos que realmente vinculan al periodista con su oficio" (Samper, 2012).

Agrega que la decisión de elevar el periodismo al rango de Comunicación Social implicó que no se produjeran ni comunicadores, ni periodistas, ni publicistas, ni relacionistas, sino comunicólogos, "una gente tan experta en Comunicación Social que de lo único que podía vivir era de enseñar Comunicación Social. Estas universidades fabricaban, entonces, profesores de Comunicación Social, ninguno de los cuales era capaz de escribir una noticia" (Samper, 2012).

Desde 1981, la Federación Latinoamericana de Facultades de Comunicación Social (Felafacs) intentó poner orden en este campo, con el deseo de trazar un mapa de ruta para los procesos formativos en Comunicación en el continente.

El proceso de Felafacs, de acuerdo con Godoy (2006), ha registrado debates en cuestiones tales como perfiles profesionales difusos o en conflicto, proyectos formativos indefinidos, apertura desmedida de programas de formación, teoricismo, formación generalista sin dominio del oficio, prácticas desarticuladas del entorno social, perfiles polivalentes, reducción de la comunicación a medios y de los medios a tecnología, lectura de necesidades sociales desde la lógica del mercado y la escasa predictibilidad de los espacios profesionales, entre otros asuntos.

Mellado (2010) precisa que "las escuelas de periodismo y las facultades de comunicación hoy se enfrentan a la dificultad de unir de manera armoniosa el contenido de las técnicas periodísticas, con las bases teóricas y el estudio de la comunicación social" (p. 277). Considera que persiste una "indefinición" que ha llevado a una tensión expresada por varios académicos latinoamericanos en el sentido que algunos consideran que "la incorporación de los estudios de comunicación ha dañado la identidad del periodismo". Otros, en cambio, aseguran que "ha sido justamente la comunicación la que le ha dado al periodismo un estatus de profesión y no de oficio" (p.279).

Advierte también que la academia latinoamericana "sigue pensando el ejercicio y significado profesional del periodismo a partir de los medios de comunicación" (Mellado, 2010, p.274),

En cuanto a los modelos que han prevalecido en la enseñanza del periodismo en Occidente y particularmente en América Latina, Martínez (2010) menciona la escuela norteamericana, portadora de una tradición fundamentada en lo pragmático que "presenta los hechos de manera básicapero con apego al rigor del dato y del hallazgo" (p. 104). También se refiere a la británica que "separa los hechos de las opiniones con un estilo sobrio que busca entregar elementos a los ciudadanos para que tomen mejor sus decisiones" (p.104). Sin embargo, y coincidiendo con Tomás Eloy Martínez, destaca el aporte latinoamericano con su énfasis literario en géneros como la crónica.

Interpretando a Martínez (2010), la convivencia de estos tres modelos se expresa en algunas metodologías como el rigor en la investigación periodística (modelo norteamericano), el interés por las humanidades y la sociología (modelo europeo) y la exploración del estilo literario en la narración de historias (modelo latinoamericano).

La Sociedad Interamericana de Prensa (SIP) también se interesó por el tema de la formación en periodismo en el continente y desde 1995 echó las bases de lo que después se convertiría en un sistema de acreditación internacional para los programas universitarios con la participación de académicos y profesionales de los medios de comunicación como pares evaluadores. La SIP creó el Consejo Latinoamericano de Acreditación de la Educación en Periodismo (Claep) con la idea de "promover una capacitación más práctica, profesional y multidisciplinaria" (Claep, 2011, p. 6)

En un primer diagnóstico de la situación de los programas de periodismo, el Claepllamó la atención sobre aspectos tales como el problema de la falta de enseñanza práctica, ${ }^{1}$ el énfasis excesivo en clases magistrales (tipo conferencia) y aprendizajes mecánicos, los planes de estudio rígidos sin posibilidades de intercambio; el número excesivo de egresados para la demanda laboral existente; las distintas posiciones en cuanto a la obligación de acreditar un título académico para ejercer como periodista y el desbalance entre la proliferación de alternativas privadas frente a las universidades públicas en cuanto a la oferta de programas en periodismo (Claep, 2011, p.7)

El Claep opera desde el año 2000 y hasta la fecha ha acreditado 15 programas de periodismo en Latinoamérica (cinco en Colombia, tres en Chile, dos en México, dos en Perú, uno en Argentina, uno en Paraguay y uno en Ecuador). Su metodología recoge la experiencia que desde 1945 viene aplicando en Estados Unidos el Consejo de Acreditación para la Educación en Periodismo y Medios de Comunicación Masiva (Acejmc).

Como se dijo anteriormente, esta iniciativa de la SIP busca acercar las propuestas formativas de las universidades latinoamericanas a las demandas de los medios, como lo señala específicamente el Manual de Acreditación del Claep (Claep, 2011, p.7).

En una nueva incursión en el tema de la enseñanza del periodismo, la Unesco convocó en diciembre de 2005 a una reunión de 20 experimentados educadores en el campo del periodismo de Asia, África, Europa, Norteamérica y Suda-
1 El Claep (2011) propone una formación que incluya un $60 \%$ en Humanidades y Ciencias (historia, derecho, ciencias económicas, ética y teoría de la Comunicación) y un $40 \%$ en medios, técnicas y lenguajes en periodismo. También recomienda un conocimiento adecuado del inglés ( $p$.21) 
2 De acuerdo con el experto de la Unesco, Hara Padhy, en Latinoamérica se ofrece una formación de corte más general a los estudiantes de Periodismo. Esto también fue advertido por Godoy (2006) en el diagnóstico que realizó sobre los debates que ha suscitado la Comunicación en la región al hacer un balance de los 25 años de Felafacs. mérica con el fin de construir un Plan Modelo de Estudios de Periodismo. Este modelo fue presentado oficialmente en el Congreso Mundial sobre Enseñanza del Periodismo realizado en 2007 en Singapur.

El Plan Modelo de Unesco plantea -siguiendo la tradición norteamericana y europea- una formación en periodismo combinada con otras disciplinas a fin de abrir más posibilidades a la reflexión crítica y al análisis de contextos. ${ }^{2}$ El Modelo aplica para cursos de licenciatura, maestría y diplomatura. Para cada nivel se establecen distintas proporciones en los componentes de formación en periodismo, otras disciplinas y ciencias humanas y sociales (Unesco, 2007). En términos de competencias, el Modelo busca desarrollar en el estudiante:

- La capacidad de redactar con claridad y coherencia valiéndose de procedimientos narrativos, descriptivos y analíticos.

- El conocimiento de instituciones políticas, económicas, culturales, religiosas y sociales nacionales e internacionales.

- El conocimiento de asuntos y cuestiones de actualidad y nociones generales de historia y geografía (Unesco, 2007, p.10).

En síntesis, el nuevo planteamiento de la Unesco (2011) en materia de formación de periodistas se enfoca en que "el estudiante tenga un conocimiento general, es decir, que no tenga sólo destrezas periodísticas sino también una sólida cultura general y una educación interdisciplinaria" (p.8), de acuerdo con lo expresado en el informe final de la Reunión Latinoamericana de Consulta sobre el Plan Modelo de Estudios de Periodismo que deliberó en Loja, Ecuador, en julio de 2011, con la participación de 14 universidades de la región, la Fnpi y el Claep, entre otros.

Cabe señalar que en esta propuesta es importante la participación de los medios de comunicación a través de convenios que permitan la realización de prácticas semestrales sobre el terreno, así como el diseño de cursos electivos que profundicen en algunas ramas del periodismo. Se promueve con esta propuesta un camino hacia la especialización en campos como la política, la economía, el arte y la cultura, las relaciones internacionales, las ciencias y la tecnología (Unesco, 2011). El Plan Modelo de la Unesco ha sido aplicado en 65 instituciones de 54 países, según los reportes vigentes a julio de 2011.

Más allá de los nuevos entornos y tensiones en los que se desarrolla el periodismo, se mantiene el debate sobre aquello que debería saber alguien que pretenda formarse en el oficio. Raúl Fuentes Navarro retoma el debate por el papel de la comunicación y en Manrique (2009), afirma que las preguntas por este campo han cambiado y a "nadie le interesa responderlas, pues hoy las miradas se vuelcan a otros referentes como la acreditación o las competencias" (p.86).
Agrega que para establecer el estado de la comunicación hoy debe observarse el desempeño de los egresados. "Hablamos quizás de cerca de 1.500 figuras profesionales que tienen que ver con producción en muchos formatos" (p.87).

Llama también la atención sobre lo que en su concepto debe replantearse en la formación de comunicadores al señalar que "hay que retomar los ingredientes básicos de la interacción del sujeto con su entorno, es decir, la capacidad de hablar, escuchar, leer y escribir" (p.87)

\section{EL TEJIDO DE UN OFICIO}

Carlos Monsiváis, en CAF (2008), recuerda que a lo largo del siglo XX la prensa no dispuso de grandes teorías que le sirvieran de orientación y fue el trabajo mismo de los periodistas el que hizo las veces de corpus teórico y modelo de formación. "Hasta fechas recientes hay tradiciones de prensa y visiones canónicas, basadas en los clásicos del periodismo o en la experiencia a secas" (p.109)

Guevara (2002) citado por Vallejo (2005) recuerda que a finales del siglo XIX y comienzos del siglo XX, la reportería en Colombia era ejercida por ciudadanos comunes y corrientes a quienes los directores de los periódicos acreditaban como reporters. Precisa Vallejo (2005) que:

La escasa profesionalización del oficio se aprecia en los periódicos de las primeras décadas [del siglo XX] porque cualquier ciudadano podía asumir el papel de reportero. Es el caso de siniestros o tragedias que terminaban por cubrir los telegrafistas, los médicos de turno o las autoridades lugareñas. (p.109)

Pena de Oliveira (2009) indica que en Francia "el periodismo suele ser ejercido por intelectuales de renombre, y arrastra una fuerte tradición cultural y política" (p.17).

El mismo autor afirma también que el periodista ha de ser un especialista, pues "a nadie le gustaría entrar en un hospital y ser atendido por un contable, o ser defendido en un tribunal por un veterinario [...]" (p.12)

Joaquín Estefanía, exdirector del diario El País de España, señala que para "enseñar a informar" se requiere un equilibrio entre conocimiento y técnica, una buena escritura y un entorno ético que obligue a las redacciones, los propietarios y los directores de los medios (CAF, 2008).

Anota además, con base en datos empíricos que maneja la FNPI, que la industria cultural de los medios de comunicación "es el sector productivo que menos dinero invierte en formación permanente de sus trabajadores" (CAF, 2008, p.27).

Periodistas colombianos como María Elvira Samper, Mau- 
ricio Vargas y Juan Gossaín fueron partidarios de replantear la formación de periodistas teniendo en cuenta que "los recién egresados están llenos de teorías y no tienen ni idea de cómo hacer un primer párrafo" y "no se puede seguir creyendo que el periodismo se aprende sentado en un pupitre" (Alba \& Buenaventura, 1997, p.18).

En dicha discusión se planteó la vía de ofrecer el periodismo a nivel de posgrado teniendo en cuenta que es más fácil enseñarles las técnicas propias del periodismo a profesionales formados en una disciplina que a un estudiante de pregrado que no lee y carece del rigor que sí garantizan otras disciplinas. "Es más fácil enseñarle a un antropólogo cómo hacer un lead o una entrevista que al otro darle el contenido, el contexto de las cosas. Las técnicas son algo accesorio, que se puede aprender después", afirmó María Elvira Samper (Alba \& Buenaventura, 1997, p.18).

Con el uso intensivo de las TIC en la labor de informar se retoma la pregunta por el lugar de la tecnología en el futuro del periodismo que formulara Germán Rey desde la FNPI al comienzo de este artículo. La socióloga Teresa Quiroz, quien por varios años ha hecho parte de la junta directiva de Felafacs, indicó en la reunión latinoamericana sobre el Plan Modelo de la Unesco que "el problema de la academia no se configura como un problema de contenidos: sería más importante ver el cómo enseñar, es decir, enfocarse en las metodologías de enseñamiento y aprendizaje" (Unesco 2011, p.8). Inclusive, se sugirieron en ese foro metodologías como la resolución de problemas y los ambientes colaborativos de aprendizaje que se articulan convenientemente a los procesos mediados por tecnología.

En este tema, Salaverría (2007) advierte que "no vaya a ocurrir que estemos formando a toda una nueva generación de profesionales con lenguajes y técnicas de un periodismo que ya no existe" (p.2). Precisa que:

Todo indica que, una vez más, la profesión periodística y la formación de los periodistas corren a velocidades diferentes. Mientras aquélla avanza rápidamente hacia un escenario de plena digitalización, ésta sigue anclada a menudo en presupuestos teóricos y prácticos que dan la espalda a todos esos cambios. (p.l)

Salaverría (2007) pone de manifiesto las resistencias que en algunos sectores de los medios generan las formas emergentes del periodismo en formato digital, frente a "la hegemonía de lo audiovisual" y la "pureza y calidad" del periodismo impreso. "Estas personas suelen ver en el periodismo de los cibermedios un paso atrás en los valores clásicos de la profesión informativa y, por tanto, tienden a menudo a despreciarlos" (p.2).
No obstante, aclara que es necesario reconocer que la mayoría de las publicaciones digitales no pueden ser consideradas como "verdadero periodismo" y muchas veces son réplicas de contenidos informativos previamente publicados por los medios tradicionales (Salaverría, 2007).

Entre sus conclusiones, el XIII Congreso de Periodismo Digital, celebrado en Huesca (España) en marzo de 2012, señala que los jóvenes no sienten nostalgia de un periodismo que no han vivido y más bien le apuestan a experimentar en escenarios que van más allá de los grandes medios.

Cuestiones como el uso de redes sociales en labores informativas y el diseño de aplicaciones para teléfonos inteligentes ganan cada día más terreno en las nuevas formas con las que el periodismo digital recompuso la vieja clasificación de medios en impresos y audiovisuales. La red funciona hoy como un nuevo ecosistema en el que conviven e interactúan formatos y plataformas en las que se mueve el periodismo.

Las redes sociales no surgieron como un medio de comunicación; no lo son. Tampoco nacieron como una herramienta para periodistas. Sin embargo, el periodismo está integrándolas a pasos agigantados como una fuente inagotable de información y como un vehículo privilegiado para hacer viajar las buenas historias hasta los lectores, estén donde estén. (Verón \& Sabes, 2012, p.10)

Las bases del oficio siguen siendo las mismas. No han cambiado los dibujos básicos de los géneros periodísticos, así la tecnología plantee formas híbridas de narración. La noticia, en esencia, se construye de la misma forma como se hacía a comienzos del siglo XX cuando las primeras agencias de noticias inventaron la pirámide invertida. La sociedad es la que ha cambiado y aquí vale la pena compartir algunas visiones.

\section{PERIODISMO SIN PERIODISTAS}

Para Ignacio Ramonet (2003) el periodismo hoy se puede hacer sin periodistas, puesto que éstos pasan a ser parte de una cadena productora de información y su papel se reduce al de simples "retocadores de despacho de agencia" (p.47).

Considera el mismo autor que "hoy, informar es esencialmente hacer asistir a un acontecimiento; es decir, mostrarlo, pasar al estadio en que el objetivo consiste en decir que la mejor forma de informarse es hacerlo directamente" (p.49).

Explica que se ha transformado el triángulo acontecimientomediador-ciudadano, en el que el acontecimiento era relatado por el mediador, quien lo filtraba, analizaba y lo hacía repercutir en el ciudadano. Hoy el triángulo se ha transformado en un solo eje en el que el acontecimiento va directamente al 
ciudadano gracias a que la cámara, el micrófono o el teléfono celular, por sí mismos, ponen en contacto directo el hecho con el público. "A mitad de camino ya no hay un espejo sino simplemente un cristal" (p.49).

Kapuscinski (2003) retoma el concepto de cadena productora de información y establece una diferencia entre el periodista y el denominado "trabajador de los medios", que ya no pone a prueba su capacidad creativa, sino únicamente su sentido de la oportunidad para sumar a la compleja industria de las noticias. Cuestiona la superficialidad y la pérdida del contacto directo con la realidad, lo que a su vez da paso al mundo virtual que vemos en la televisión o la Internet.

Afirma que la industria de las noticias domina un panorama que abarca no solo la manera como se realiza el trabajo periodístico, sino los nuevos roles que cumplen los reporteros. "La manipulación de las noticias en los medios, nos convierte a todos en prisioneros de un lenguaje reducido, pobre y limitado" (Kapuscinski, 2003, p.31).

Considera necesario retornar a las formas creativas de "contar historias" que hacen acopio de la sensibilidad, la capacidad de relacionarse con el otro y la creatividad para producir textos ricos en descripciones. Estos elementos se han perdido ante la avalancha de imágenes que hoy dan cuenta de lo que sucede en el mundo (Kapuscinski, 2003). Ramonet (2003), Kapuscinski (2003) y Monsiváis (2008) coinciden en que "ver no es comprender" en un modelo informativo dominado por la imagen y el rating.

Por su parte, Tomás Eloy Martínez (2000) reivindica la narración como medio para recuperar las bases del periodismo. Considera que los diarios subsistirán en el siglo XXI si logran mantener el equilibrio entre el modelo tradicional de las noticias (pirámide invertida), el manejo de información con antecedentes y contexto y la posibilidad de construir historias contadas por eficaces narradores.

Recuerda que el periodismo en escenarios como América Latina está ligado al hecho de "contar historias" y hace una relación de ejemplos de escritores que fueron reporteros (Martí, Vargas Llosa, García Márquez, etc.). Sin embargo, advierte acerca de las dificultades que hoy existen para rescatar géneros como la crónica y el reportaje que han desaparecido de los grandes medios.

El periodista no es un agente pasivo que observa la realidad y la comunica; no es una mera polea de transmisión entre las fuentes y el lector sino, ante todo, una voz a través de la cual se puede pensar la realidad, reconocer las emociones y las tensiones secretas de la realidad, entender el por qué y el para qué y el cómo de las co- sas con el deslumbramiento de quien las está viendo por primera vez. (Martínez, 2000, p.43)

\section{PRENSA Y SOCIEDAD}

Kovach \& Rosintiel (2003), citados por Pena de Oliveira (2009) sitúan el modesto origen de la prensa moderna en las tabernas y cafés de Londres y París donde se escuchaban los relatos de los viajeros a comienzos del siglo XVII

...Allí los dueños de los pubs (casas públicas) estimulaban las conversaciones con los viajeros, pidiéndoles que contasen lo que habían visto por el camino. En Inglaterra había cafés especializados en informaciones específicas. Los primeros periódicos habían salido de esos cafés hacia 1609, cuando los tipógrafos más atrevidos comenzaban a recopilar informaciones, chismorreos y discusiones políticas en los propios cafés, imprimiéndolo todo más tarde. (Pena de Oliveira, 2009, p.27)

Javier Darío Restrepo, exdefensor del lector del diario El Tiempo, de Bogotá, ex columnista (censurado) de El Colombiano, de Medellín, y quizás el más importante especialista en los temas de ética periodística en Colombia en los últimos años, toma esta misma referencia de Kovach \& Rosintiel (2003) para señalar cómo desde estas conversaciones de café se construye un sentido de sociedad ligado al nacimiento de la prensa.

En esas conversaciones de café no se hizo la Revolución Francesa, pero es probable que sin esos intercambios de ideas, la Revolución hubiera tomado otros rumbos o hubiera retardado su entrada en la historia. Todos esos periódicos, lo mismo que la Enciclopedia de Diderot, aclimataron las ideas revolucionarias en la conciencia de los franceses, que es la otra forma como los periódicos hacen sociedad: estimulando el pensamiento, promoviendo reflexiones, convirtiendo a los lectores en participantes activos en los procesos de cambio de la sociedad. (Restrepo, 2004, p.2)

Restrepo desarrolló el concepto de la "prensa como tejedora de sociedad”, en una conferencia dictada en Medellín en 2004 organizada por el periódico El Pulso. Señaló:

El poder de leer el futuro, que antes se les dejaba a videntes, horóscopos y manipuladores de cartas, hoy lo racionalizan la prospectiva y el buen periodismo que orienta a las sociedades en la exploración de lo posible. Ir mas allá de lo que muestran los sentidos, investigar las causas de los hechos y preguntarse por lo posible, es otra forma del poder de los medios. En toda realidad hay una dimensión que se mantiene invisible hasta que alguien la visibiliza: es lo posible. Ese periodismo que estimula el progreso 
de las sociedades, que en cada pieza de comunicación riega semillas de entusiasmo, que mantiene un alto nivel espiritual de iniciativa, es el que pone el poder de lo posible al servicio de las comunidades. Es el periodismo que está difundiendo esperanza porque, aún en el relato de los más sombríos episodios, insiste en buscar salidas y en hallar propuestas. (p.15)

Por su parte, Fabio López de la Roche (2003) plantea la incomprensión que ha existido desde las Ciencias Sociales hacia el periodismo, al punto que desde la academia se señala a éste como "un saber de tercer o cuarto orden, que no alcanzaría la decantación y la pureza científica del saber categorial y conceptual de las disciplinas sociales" (p.10).

Muchas investigaciones en el campo social se apoyan en los registros de prensa, aunque López de La Roche (2003) hace una aclaración en cuanto al alcance que tienen las referencias de prensa en el marco de una investigación:

Los historiadores, por ejemplo, suelen trabajar con la prensa escrita como una importante fuente documental para la reconstrucción de hechos, períodos y comportamientos de personajes en la historia. Pero es muy probable que en la aproximación crítica a sus fuentes el historiador olvide, por simple desconocimiento, que esas noticias y otros géneros periodísticos, son no sólo representaciones parciales y fragmentarias de la realidad histórica por ellas reconstruida, sino que adicionalmente, ellas han sido escritas desde ideologías profesionales (ideologías del periodismo acerca de qué es noticia, que sería lo noticiable y lo que le interesaría a la gente en distintos momentos históricos), así como desde ciertas rutinas ocupacionales que caracterizan el funcionamiento del periodismo como profesión (privilegio del hecho espectacular y de informaciones de connotaciones negativas por sobre las positivas o constructivas, entre otras). (p.11)

Sobre el periodismo y el ejercicio periodístico existen percepciones sociales que lo sitúan entre lo culto y lo vulgar o entre el encomiable papel de fiscalizador de los poderes instituidos (cuarto poder) y la fuente de muchos males derivados del eterno afán por inmiscuirse en lo más sórdido de la naturaleza humana.

No obstante, al mostrar los contrastes en cuanto al talante de la prensa europea, Pena de Oliveira (2009) afirma que "en Inglaterra abundan periódicos de larga tradición, es el caso de The Times; lo que no es óbice para que los tabloides ${ }^{3}$ tengan una fuerte presencia. Y es que la isla de Su Majestad es el paraíso de los paparazzi" (p.17).

\section{VALORES Y CREENCIAS EN DISCUSIÓN}

Desde cuando aparecieron los programas universitarios en periodismo, la discusión ha girado mayormente en aquello que la universidad enseña, pues muchas veces los jóvenes practicantes o que recién comienzan a ejercer el oficio se sienten huérfanos frente a cosas que no vieron en sus clases.

Una investigación de Juan Guillermo Arias, Edinson Monroy y Juan Pablo Tovar en torno a los valores, las creencias y la autopercepción del periodismo y de los periodistas en Bogotá, Colombia, revela aspectos sobre los que gira el oficio en una población mayoritariamente ubicada entre los 20 y 25 años que tiene la función de informar desde los más importantes medios de comunicación de Colombia.

Se aplicó una encuesta a 141 personas entre jefes de redacción, periodistas y editores de los siguientes medios con cubrimiento nacional: El Tiempo, Revista Semana, Revista Cambio, y diario La República, en lo que tiene que ver con prensa; $R C N$ básica, La Wy Caracol básica, en radio; Noticias RCN, Caracol Noticias, Noticias Uno y NTN 24 horas de RCN, en televisión.

Para los investigadores resultó significativo comprobar cómo para los periodistas el papel de la universidad en su formación tiene que ver más con el capital social que esta representa que con el capital cultural específico que imparte. Precisan que este hallazgo se sustenta en el hecho que "son muy pocos los periodistas que indican haber participado en concursos abiertos que les hayan exigido la superación de pruebas de conocimientos específicos" (Arias, Monroy \& Tovar, 2010, p.97).

Vale la pena señalar que ante la pregunta abierta que se les formuló a los encuestados sobre "¿Cuándo cree usted que nació el periodismo?" el 40\% asocia los inicios de la profesión con los comienzos de la comunicación y con acontecimientos tan difusos e incoherentes como "el periodismo nació en la Edad Media con los cronistas de Indias" o "cuando el arcángel San Gabriel comunicó a María su embarazo” (p.92).

Otra de las conclusiones del estudio muestra cómo los encuestados le dan más valor a las materias prácticas (talleres), mientras que los jefes de redacción señalan "el olfato periodístico" como criterio de selección de un redactor.

En cuanto a la autopercepción del prestigio social del periodista en comparación con otras profesiones, la investigación mostró cómo los mismos periodistas se colocan en el puesto 14, por debajo de los médicos, abogados, publicistas, economistas, ingenieros civiles, pilotos, psicólogos, arquitectos, artistas, sacerdotes, ingenieros de sistemas, diseñadores gráficos y sociólogos. Consideran que el periodista estaría por encima de los profeso-
3 Los tabloides europeos son considerados mayoritariamente una prensa liviana que se ocupa de temas superficiales y en algunos casos de escándalo. Basta recordar la nube de fotógrafos (paparazzis) que perseguía a la princesa Diana de Gales por las calles de París, el 31 de agosto de 1997, cuando perdió la vida junto con su amigo Dodi Al Fayet. 
res universitarios, los políticos, los profesores de bachillerato, los agricultores y los taxistas.

Ryszard Kapuscinski (2003), el emblemático reportero polaco, recuerda que hace medio siglo el oficio de periodista se catalogaba de manera muy diferente a lo que ocurre hoy:

Se trataba de una profesión de alto respeto y dignidad, que jugaba un papel intelectual y político. La ejercía un grupo reducido de personas que obtenían el reconocimiento de sus sociedades. Un periodista era una persona de importancia, admirada. Cuando andaba por la calle, todos lo saludaban. (p.13)

El carismático jefe de redacción del periódico El Espectador, de Bogotá, José Salgar (1999), recuerda la prensa en el siglo XX en Colombia como la cuna de donde emergieron destacados intelectuales y líderes, algunos de los cuales inclusive llegaron a ser presidentes de la República.

Los diarios escritos fueron en esa época las primeras y únicas escuelas de periodismo para una generación de intelectuales y artesanos de la imprenta que resultó muy brillante y surtió de dirigentes no sólo a la incipiente industria editorial sino a diversos sectores públicos y privados". (p.30)

\section{ALGUNAS APUESTAS EN LA FORMACIÓN DE PERIODISTAS}

Pensar el periodismo en una perspectiva formativa no es cosa de juntar piezas para aproximarse a las competencias que debería tener un sujeto sobre el que recae la tarea y la responsabilidad de informar. En la transición hacia el nuevo siglo, Salgar (1999) escribió que "la universidad tendrá el doble papel de formar a periodistas en áreas o disciplinas específicas y de especializar en periodismo a profesionales de áreas diferentes" (p. 6).

Pena de Oliveira (2009) formula una serie de preguntas acerca de la formación del periodista que pueden ayudar a pensar la cuestión:

... Para ser periodista es preciso estudiar periodismo, y eso es algo que sólo se hace en la Universidad. Pero la cuestión es ¿cuál es la formación ideal en las facultades de Periodismo? ¿Las disciplinas 'técnicas' como la Redacción Periodística y el Periodismo televisivo deben ser privilegiadas? ¿O, por el contrario, el curso debe tener un carácter reflexivo haciendo énfasis en las llamadas disciplinas teóricas? ¿Y cuál ha de ser la formación de los profesores? ¿Profesionales con experiencia en el oficio o doctores con un gran historial académico? ¿Los equipamientos y las instalaciones son fundamentales? ¿Cómo debe ser la estructura pedagógica? (p.12)

El mismo autor recoge el análisis que sobre este tema hiciera el director de la Escuela de Periodismo de la Universidad de
Columbia, Nicholas Leman, en 2003 y que el New York Times publicó en su edición del 14 de mayo de 2003:

Una de las propuestas específicas de Leman es la creación de un curso que examine las diferentes formas de 'buscar la verdad', además de abordar los diferentes conocimientos exigidos en áreas como Derecho, Economía, Psicología, Estadística y Filosofía. También sugirió que los alumnos de primer año hicieran cursos sobre Literatura Clásica y grandes pensadores. En el segundo año los alumnos se especializarían es una disciplina concreta, como pueden ser Ciencia, Religión o Economía, y producirían una publicación semejante a la que hacen los alumnos de las facultades de Derecho americanas". (Pena de Oliveira, 2009, p.16)

En los nuevos entornos digitales, Salaverría (2007) fundamenta su apuesta formativa en dos pilares: la innovación y la renovación.

La innovación corresponde a la exploración de nuevos lenguajes y modelos editoriales específicos del ciberespacio. Por su parte, la renovación comprende la actualización de todas las disciplinas periodísticas clásicas a la luz de las mudanzas propiciadas por el impacto digital en la profesión. El reto consiste en formar periodistas multiplataforma que, además de conocer y respetar los valores clásicos del periodismo -honestidad, imparcialidad, espíritu crítico...-, estén preparados para cumplir con su quehacer profesional en cualquier medio, ya sea impreso, audiovisual o interactivo. (p.4)

En la discusión sobre la formación de periodistas no puede dejarse de lado el papel que cumplen el docente y las metodologías de aprendizaje, aunque esto último merecería un capítulo aparte y un análisis más extenso.

La Reunión Latinoamericana de Consulta sobre el Plan Modelo de Estudios de Periodismo de la Unesco de 2011 llamó la atención acerca de la falta de cursos de maestría y doctorado en periodismo en América Latina, circunstancia que no ha contribuido a elevar la calidad de la formación que hoy se imparte en las Facultades de la región.

Los docentes de periodismo provienen en su mayoría de los medios de comunicación, pero no han tenido ni el tiempo ni los recursos suficientes para realizar un posgrado. En este sentido, la reunión sobre el Plan Modelo de la Unesco propone la apertura de más maestrías en periodismo que permitan formar más profesores que después impartan cursos a nivel de posgrado. La idea es aumentar el número de periodistas con maestría. 


\section{UN CONOCIMIENTO ESPECIALIZADO}

Desde el punto de vista del desarrollo curricular, la Universidad Complutense de Madrid abordó desde 1975 la cuestión del periodismo como un saber especializado, cuando comenzó a impartir la asignatura "Información Periodística Especializada" en el plan de estudios de la Facultad de Ciencias de la Información (Fernández del Moral, 2004, p.20). Señala que se trataba de caracterizar la información periodística dentro del modelo de Otto Groth (actualidad, periodicidad, universalidad y difusión) pero con algunas especificidades.

En este escenario la información es vista, analizada y trabajada desde tres niveles. El primero relacionado con los contextos sociales, políticos y jurídicos; el segundo, con la estructura y manejo de los medios y el tercero, con las particularidades del mensaje.

Fernández del Moral (2004) explica que el primer nivel es visto como una macroestructura que fija las condiciones que cada sociedad imprime al ejercicio del periodismo. En esta mirada intervienen saberes de los campos económico, político y jurídico. En el segundo nivel aparecen algunas asignaturas típicas de la Comunicación Social como el análisis de medios y los estudios de audiencias, junto con otras herramientas como la estructura de medios, el pluralismo informativo o el carácter multimedia de las casas productoras de información. En el tercer nivel, el autor establece tres microestructuras: una sintáctica, una semántica y otra pragmática, que se relacionan a su vez con el estudio de la forma, el código y el contenido. Precisa que la forma se contempla en asignaturas relacionadas con tecnologías de la información, mientras que el código se ve en la redacción periodística, en tanto que el contenido "aparece ya como un modo concreto de tratar la realidad, respondiendo al qué de la información periodística" (p.22).

Desde estos tres niveles, el investigador precisa que se puede dar "un punto de unión entre el conocimiento especializado y la opinión pública, entre el trabajo de los expertos y la sociedad" (p.22). No obstante, llama la atención sobre lo difícil que resulta dar cuenta de un conocimiento especializado con las actuales estructuras de las licenciaturas o programas de periodismo. "La formación específica de cada especialidad queda reservada al posgrado" (p.31).

Cabe aclarar que sobre el asunto de la especialización existen distintos enfoques. Una de esas miradas parte de la organización que los medios dan al cubrimiento de los distintos temas. Sobre esto, Samper (2012) afirma que antes que un especialista en deportes, economía o política, el periodista debe tener un dominio mínimo del oficio para poder moverse en el universo informativo.

Yo creo que conviene una especialización, pero no una especialización que impida ejercer el periodismo en general. No se puede pedir que todos sepan absolutamente de todo. Que averigüen sí, pero que sepan todo, no. Es conveniente una especialización pero no hasta el punto que se niegue la función esencial del periodismo. Para cubrir una puñalada no se necesita ser especialista...

\section{ENCUENTRO ENTRE TEORÍA Y PRÁCTICA}

La tensión entre teoría y práctica en la enseñanza del periodismo ha llevado a algunas universidades y reconocidos medios de comunicación a realizar alianzas.

En 1985, el diario El Clarín de Buenos Aires y la Universidad Católica de Argentina (UCA) abrieron de manera conjunta el Curso Superior de Perfeccionamiento en Periodismo, orientado a egresados de las carreras de periodismo de todo el país. Se seleccionaban 20 jóvenes, quienes durante seis meses recibían cursos en la UCA y desarrollaban su pasantía en las distintas secciones del periódico. Hoy este programa no se está ofreciendo.

En 1986, el Grupo Prisa -propietario, entre otros medios, del diario El País de España- y la Universidad Autónoma de Madrid crearon un modelo de formación posgradual orientado a licenciados en cualquier disciplina. La propuesta se desarrolla tanto en la universidad como en los medios del conglomerado mediático. Se otorga el título de maestría y de acuerdo con la información publicada en la web: "el objetivo de la Escuela es ofrecer una formación que permita a sus alumnos, sea cual fuere su carrera universitaria, ejercer un periodismo riguroso en cualquier medio de comunicación, ya sea impreso, digital o audiovisual" (Escuela de Periodismo UAM-El País, 2012).

Por su parte, la Universidad de Columbia, en Estados Unidos, ofrece un modelo similar en su Master of Science Degree, tomando a la ciudad de Nueva York como el gran laboratorio para desarrollar historias en distintos formatos que posteriormente son publicadas en revistas, programas de televisión, radio y portales vinculados al proyecto (Columbia Journalism School, 2012).

De igual forma, la Escuela de Periodismo de la Facultad de Comunicaciones de la Universidad Católica de Chile y el diario El Mercurio se aliaron desde 2004 para ofrecer un magíster en periodismo escrito, compartiendo espacios formativos y de práctica tanto en la institución educativa como en las instalaciones del diario. El programa tiene una duración de 12 meses (tiempo completo) y se ofrece tanto a 
reporteros en ejercicio como a personas provenientes de las áreas de humanidades, las ciencias y las artes. Es dictado por reconocidos periodistas e incluye una pasantía de medio tiempo y un proyecto final.

Otra experiencia es la referida por Pena de Oliveira (2009) en la Universidad Federal Fluminense de Brasil, a través de alianzas con medios universitarios, comunitarios y la gran prensa como Journal de Brasil y O Dia para la circulación de materiales realizados por los estudiantes del programa de Comunicación Social-Periodismo. El factor diferenciador radica en el papel de los docentes, encargados "de avanzar en los formatos y en el contenido, evitando las fórmulas de los telediarios tradicionales" (Pena de Oliveira, 2009, p.14)

A partir de 2011, la Universidad del Rosario y Publicaciones Semana, en Colombia, replicaron este modelo ofreciendo una maestría en Periodismo de tres semestres de duración y un diseño curricular que incluye diez asignaturas directamente relacionadas con el oficio y la investigación periodística. El primer semestre se cursa en la sede de la universidad, el segundo en los entornos de los portales de las publicaciones del grupo Semana y el último en las redacciones de las revistas Semana, Dinero y Soho.

\section{A MANERA DE CONCLUSIÓN}

El panorama descrito en este artículo no cambia si no se modifica la forma como se está enseñando el periodismo. Para esto, es necesario formar mejor a los profesores con el fin de torcer la tendencia a reproducir los esquemas de "periodismo sin periodistas" (Ramonet, 2003 y Kapuscinski, 2003). Las universidades deben acercarse a los medios y ofrecer cursos de especialización a los reporteros con intereses en la docencia, facilitando horarios y ofreciendo costos asequibles, tal como lo planteó la reunión de Loja (Ecuador) en torno al Plan Modelo de Estudios de Periodismo de la Unesco. A quienes han abandonado la reportería y hoy se encuentran impartiendo cátedras universitarias, cabría la posibilidad de interesarlos en maestrías y doctorados en periodismo para profundizar en la investigación y quizás apostarle fuertemente al afianzamiento de un corpus teórico para el periodismo más allá de los estudios de recepción o el Análisis Crítico del Discurso (ACD). Se requieren, como dice la Unesco, más profesores de periodismo que ayuden a formar profesores de periodismo.

Lo anterior facilitaría el diálogo necesario entre el Periodismo y las Ciencias Sociales que reclama López de La Roche
(2003) favoreciendo una mejor contextualización de la información y el descubrimiento de nuevas facetas en los objetos de investigación.

La tensión teoría-práctica, incluida tanto en los diagnósticos de Felafacs, el Claep y la FNPI como en las percepciones de los periodistas en ejercicio, implica el rediseño de esquemas curriculares que conecten más los contenidos programáticos con la realidad. Las asignaturas deberían desarrollarse paralelamente con los grandes temas de la actualidad local, nacional o mundial y para eso es necesaria nuevamente la llave universidad-medios en la generación de espacios tanto de aprendizaje como de circulación de información mediante convenios interinstitucionales como los que opera la Universidad Federal Fluminense de Brasil.

El periodismo especializado encuentra en el modelo desarrollado por la Universidad Complutense de Madrid un buen referente, si tiene en cuenta que el proceso que va del contexto (la macroestructura) al mensaje (la microestructura) hace participar al estudiante de diversos saberes que enriquecen la visión de los acontecimientos que se comunican. Ante la creciente oferta de programas de Comunicación, la especialización puede marcar importantes diferencias y mejorar la calidad informativa, sin olvidar, por supuesto, las competencias básicas que debe tener todo buen reportero en una región del mundo donde, como lo señalan Carlos Monsiváis y Tomás Eloy Martínez, existe una importante tradición de buenos narradores que se formaron en los periódicos.

El periodismo se ha descentrado en los últimos años de la Comunicación Social y hoy interesa a profesionales de otras disciplinas que han visto en él una forma de ejercicio profesional. Es necesario, por tanto, seguir desarrollando propuestas de posgrado para este segmento de aspirantes.

La tecnología se inserta hoy en el terreno de nuevas experiencias narrativas que es necesario explorar con la idea de enriquecer el relato periodístico y, como señala Salaverría (2007), es necesario desarrollar competencias para contar historias en varias plataformas (texto, multimedia, televisión, fotografía, redes sociales, dispositivos móviles).

Las propuestas formativas, las lecturas recíprocas entre sociedad y prensa, los nuevos modelos informativos y las discusiones de los periodistas y los académicos sobre el oficio nos retornan a la pregunta movilizadora de la FNPI ¿Hacia dónde va el periodismo?, para comprobar que aún no hay una respuesta contundente, solo apuestas.

Pero acaso ipuede hacerse algo distinto? Javier Darío Restrepo, en CAF (2008) recoge una metodología de trabajo desa- 
rrollada en los talleres de la FNPI, inspirada en el llamado "periodismo de propuesta", planteado por Geraldinho Vieira, en Cartagena, Colombia, en 2000.

El ejercicio parte de una pregunta retadora en torno a la manera como tradicionalmente se cubren las catástrofes: “¿Puede hacerse algo distinto?”. De allí surgieron propuestas encaminadas no solo a recopilar la información necesaria para dar cuenta del acontecimiento, sino también a profundizar en una investigación que permita conocer más a fondo las causas y a su vez haga posible la búsqueda de propuestas para evitar la repetición de las consecuencias del desastre.

Para el escenario descrito anteriormente se requieren periodistas formados no solamente en las competencias de narrar o contar, sino también de profesionales formados para poder interpretar de la mejor forma posible el escurridizo pedazo de realidad que se examina desde la información; en otras palabras, profesionales capaces de leer y narrar la sociedad que les tocó vivir en el siglo XXI.

\section{REFERENCIAS}

Alba, G. \& Buenaventura. J. G. (1997). Facultades de Comunicación: en el ojo del huracán. Signo y Pensamiento, 16(31), 11-24.

Arias, J.G., Monroy, E. \& Tovar, J.P. (2010). El periodista frente a su formación. Una aproximación a los programas de comunicación social desde la cultura y los valores en el campo periodístico bogotano. Signo y Pensamiento, 29(56), 87-101.

CLAEP (2011). Acreditación en Periodismo en América Latina. Principios, políticas y mecanismos de Acreditación Recuperado de http://www.claep.org/Manual_de_ Claep_2011.pdf

Columbia Journalism School (2012). Master of Science Degree. Recuperado de http://www.journalism.columbia.edu/ page/85-master-of-science-degree/85

Escuela de Periodismo UAM-El País (2012). Objetivos. Recuperado de http://escuela.elpais.com/objetivos.html

Godoy, A. M. (Septiembre, 2005). Estado del arte en la formación de comunicadores en América Latina. Trabajo presentado en el XXIII Encuentro Académico de AFACOM Medellín, Colombia.
Kapuscinski, R. (2003). Los cinco sentidos del periodista. México, D.F.: Fondo de Cultura Económica.

López de la Roche, F. (2003). El periodismo: ese relegado objeto de estudio y debate ciudadano. Diálogos de la Comunicación, 66, 10-15.

Manrique, J. (2009). Comunicación es cualquier cosa. Comunicación, 148, 86-88.

Marques de Melo, J. (1997). Enseñanza del periodismo en América Latina. Singularidades del modelo brasileño. Signo y Pensamiento, 26(31), 123-136.

Martínez, T. (2000). Periodismo y narración, desafíos para el siglo XXI. El Malpensante, 27, 37-44.

Martínez, A. (2010). Periodismo cognitivo como estrategia en el aula de clase. Signo y Pensamiento, 29(56), 102-112.

Mellado, C. (2010). La voz de la academia: reflexiones sobre periodismo y comunicación. Signo y Pensamiento, 29(56), 274-287.

Pena de Oliveira, F. (2009). Teoría del Periodismo. México, D.F.: Alfaomega. 
Ramonet, I. (2003). La tiranía de la comunicación. Barcelona, España: Mondadori.

Restrepo. J.D. (Octubre, 2004). La prensa tejedora de sociedad. Trabajo presentado en el Seminario de Comunicación sectorial y científica construcción de sociedad a través de la información, Medellín, Colombia.

Salaverría, R. (2007). Formar periodistas en la era de Internet. Recuperado de http://dspace.unav.es/dspace/ bitstream/10171/7379/1/formar_periodistas_en_la_era_ de_internet0001.pdf

Salgar, J. (1999). Coletilla al fin de siglo. Bogotá, Colombia: Universidad Sergio Arboleda.
Samper, D. (2012). De la P a la O. Lo esencial del periodismo. Recuperado de http://reddeperiodismocultural.fnpi.org/ de-la-p-a-la-o-lo-esencial-del-periodismo-segun-danielsamper-pizano-charla-en-vivo-1100-col

Unesco (2007). Plan Modelo de Estudios de Periodismo. Recuperado de http://www.comiunesco.org.pe/doc/10/ plan_estudio.p

Unesco (2011). Reunión Latinoamericana de consulta sobre el Plan Modelo de Estudios de Periodismo de la UNESCO. Informe Final. Recuperado de abelsuing.files.wordpress. com/2011/08/informe-final.docx

Vallejo. M. (2005). Cuando los periodistas colombianos salieron a la calle. Signo y Pensamiento, 25(48), 106-122.

\section{SOBRE EL AUTOR}

Jorge Manrique Grisales es Comunicador Social-Periodista de la Universidad de La Sabana (Colombia); Magíster en Tecnologías de la Información aplicadas a la Educación de la Universidad Pedagógica Nacional (Colombia); Especialista en Informática para la docencia de la Universidad Central (Colombia). / Académico de la carrera de Comunicación de la Pontificia Universidad Javeriana Seccional Cali. / Investigador de la línea de Comunicación y Educación del grupo de investigación de Procesos y Medios de la Facultad de Humanidades y Ciencias Sociales de la Pontificia Universidad Javeriana de Cali, Colombia. Dirección: Av. Cañasgordas, Calle 18 No 118-250, Cali, Colombia. Mail: jmanrique@javerianacali.edu.co

-Forma de citar este artículo :

Manrique, J. (2012). Enseñar periodismo para leer y narrar la sociedad del siglo XXI, Cuadernos de Información, 30, xx-xx 